\title{
COMPLICACIONES MATERNO NEONATALES EN EL HOSPITAL FÉLIX MAYORCA SOTO - TARMA - 2017
}

\author{
Neonatal maternal complications at the Félix Mayorca Soto Hospital - Tarma - 2017 \\ Vilma Eneida Palpa Inga, ${ }^{1, a, b}$, Nelly Marleni Hinostroza Robles ${ }^{2, c, d}$
}

\begin{abstract}
${ }^{1}$ Escuela de Obstetricia. Universidad Nacional Daniel Alcides Carrión, Filial Tarma .Junín, Perú
${ }^{2}$ Escuela de Enfermería. Universidad Nacional Daniel Alcides Carrión, Filial Tarma. Junín, Perú.

a Obstetra, ${ }^{b}$ Magister en Salud Pública y Gerencia Sanitaria, ${ }^{C}$ Licenciada en Enfermería, ${ }^{d}$ Doctor en Ciencias de la Salud
\end{abstract}

\section{RESUMEN}

Las complicaciones maternas neonatales, en gestantes adultas y adolescentes se presentan con mayor frecuencia cuando se asocia a factores de riesgos, que pueden llegar a causar la muerte. El fin primordial de la atención materno-neonatal es evitar los daños y lograr el bienestar de las mujeres y sus hijos. Objetivo: Comparar las complicaciones materno neonatales en gestantes añosas y adolescentes atendidas en el Hospital Félix Mayorca Soto. Marzo - agosto del 2017. Material y Métodos: Estudio descriptivo comparativo de corte transversal. Resultados: Las complicaciones en el embarazo de adolescentes y adultas fueron ITU (Infecciones transuretrales) y ANEMIA. Las complicaciones del parto en las gestantes adolescentes fueron; expulsivo prolongado y retención de restos y en las gestantes adultas fueron conducción e inducción de parto y retención de restos. Conclusiones: Las complicaciones neonatales en gestantes adolescentes fueron de RCIU (Retardo de crecimiento intrauterino) y Prematurez, en gestantes adultas fueron de RCIU e Ictericia Neonatal. Palabras clave: Complicaciones, Madre - Neonato, Adolescentes y adultas

\section{SUMMARY}

Neonatal maternal complications in adult pregnant women and adolescents occur more frequently when associated with risk factors, which can lead to death, and the primary purpose of care is to avoid these damages and achieve well-being of women and their children. Objective: To compare maternal and neonatal complications in elderly pregnant women and adolescents treated at the Félix Mayorca Soto Hospital March to August 2017. Material and Methods: Cross-sectional comparative descriptive study. Results: The complications in pregnancy in both the adolescent and adult population were UTI, ANEMIA, in the complications of childbirth in the adolescents were, prolonged expulsive and retention of remains in the adult pregnant women were conduction, induction of childbirth and retention of remains. The neonatal complications were adolescent pregnancies were IUGR, and prematurity, in adult pregnant women were IUGR and Neonatal Jaundice.

Key words: Complications, Mother - Neonate, Adolescents and adults.

\section{INTRODUCCIÓN}

El embarazo en la vida reproductiva ha sido objeto de curiosidad y preocupación científica, no sólo por su alta frecuencia en los últimos años, sino por las afecciones que son propias para cada edad y la innegable repercusión que la gestación tiene para la familia y la sociedad. Cada vez es más frecuente que por razones sociales, la mujer postergue su embarazo, en función de adquirir una mejor preparación y una mejor condición económica, además de procurar una pareja estable.

Según el fondo de poblaciones de naciones unidas (UNFPA) en el 2012 el problema de embarazo adolescente en el Perú mostraba que el $13 \%$ de las mujeres entre los 15 y 19 años han salido embarazadas; el $38 \%$ de las adolescentes en el área rural que hablan 
lenguas nativas se embarazaron; 7 de cada 100 muertes por causas maternas son madres de 17 años o menos; solo el $32 \%$ de las mujeres embarazadas entre los 15 y 19 años deseaban estar embarazadas; 6 de cada 10 mujeres adolescentes no unidas y sexualmente activas usan métodos anticonceptivos modernos, $53 \%$ de los hombres adolescentes no unidos $y$ sexualmente activos usan condón fondo naciones Unidas (1).

El embarazo adolescente es un problema de salud pública debido a la conducta sexual de las jóvenes, por su precocidad en las relaciones sexuales, sin el uso adecuado de los métodos anticonceptivos.

La maternidad tardía se asocia generalmente a alteraciones preexistentes que inevitablemente se incrementa con la edad, las cuales en su mayoría se identifican como grupo de riesgo. En este período de tiempo aparecen enfermedades como la prematuridad, hipertensión arterial crónica, fibroma uterino, malformaciones congénitas y alteraciones genéticas, entre otras, que afectan sensiblemente la morbimortalidad materno infantil, ya que estas mujeres cursan un embarazo donde se observa mayor frecuencia de afectaciones médicas obstétricas que favorecen las complicaciones en el parto y aumentan las intervenciones quirúrgicas, registrándose un incremento de muertes fetales y recién nacidos $(\mathrm{RN})$ de bajo peso.

La edad en que las mujeres comienzan a buscar su primer embarazo se ha venido retrasando en las sociedades desarrolladas. Este retraso en la edad se encuentra relacionado a ciertos factores, como la necesidad de tener una condición financiera y profesional que ofrezcan seguridad, un deseo para esperar a iniciar una relación sentimental estable, segundos matrimonios y un mejor acceso a diferentes métodos de anticoncepción.

Se ha considerado al embarazo en la adolescente y en la adulta como un embarazo de alto riesgo ya que se experimenta mayor incidencia de complicaciones durante la gestación y el parto, tanto para la madre, así como mayor posibilidad de complicaciones para el recién nacido. Estas situaciones nos motivan a realizar la presente investigación y promover medidas preventivas promocionales que favorezcan el embarazo en óptimas condiciones de salud de la madre y su hijo. Señalándose como objetivo comparar las complicaciones maternas neonatales en gestantes añosas y adolescentes atendidas en hospital Félix Mayorga Soto.

\section{MATERIALES Y METODOS}

La investigación fue de tipo cuantitativo, diseño descriptivo-comparativo de corte transversal, la unidad de análisis lo constituyeron 202 gestantes con sus respectivos hijos, hospitalizados durante el periodo de estudio. Se recolecto la información a través de sus historias clínicas maternoperinatal. Para el análisis estadístico de los cuadros con datos cualitativos se hizo uso de la prueba de comprobación proporcional no paramétrica de la Ji CUADRADA.

\section{RESULTADOS}

La mayoría de las participantes tienen entre 2631 años con un 33\%, en segundo lugar entre 2025 años con un $26 \%$, en tercer lugar entre 32-37 años con un $22 \%$, en cuarto lugar entre 14-19 años con un $11 \%$ y en último lugar de 38-43 años con un $8 \%$ (Tabla № 1 ).

El estado civil de la mayoría de las participantes adolescentes su es soltera con un $74 \%$ en primer lugar, y conviviente con un $26 \%$. En cuanto a las gestantes adultas en primer lugar su estado civil es de conviviente con un $49 \%$, en segundo lugar casadas con un $22 \%$, tercer lugar son divorciadas con un $18 \%$ y en cuarto lugar solteras con un 10\% (Tabla № 2).

Las gestantes adolescentes en su mayoría son primigestas en un $74 \%$, y multigesta en un $26 \%$. En las gestantes adultas en su mayoría se evidencia que son multigesta en un $80 \%$ y primigestas en un $20 \%$ (Tabla № 3 ). 
Asimismo la mayoría de gestantes adolescentes tuvieron entre 2 a 6 atenciones prenatales en un $48 \%$, seguido entre 1 a 3 atenciones prenatales con un $39 \%$. En las gestantes adultas las atenciones prenatales recibidas son de 2 a 6 que representa un 56\%, seguido de entre 1 a 3 atenciones con un 25\%. En conclusión las gestantes todas sin excepción recibieron atenciones prenatales (Tabla № 4).

En su mayoría las gestantes adolescentes tuvieron parto distócico vaginal en un 39\%, seguido de cesáreas en un $35 \%$ y parto eutócico en un $26 \%$. En las gestantes adultas presentaron parto distócico vaginal en un $49 \%$, seguido de cesáreas en un $32 \%$ y parto eutócico $19 \%$, de los cuales se concluye que la mayoría de gestantes terminaron con un parto Distócico vaginal, por diferentes circunstancias entre adolescentes y adultas (Tabla № 5 ).

Se evidenció también que las gestantes adolescentes presentaron complicaciones en el embarazo siendo el primero de una ITU con un $26 \%$, segundo lugar Anemia con un $22 \%$ y en tercer lugar Amenaza de Parto Pre término con
13\%. En las gestantes adultas en primer lugar presentaron ITU, seguido de Anemia en un 19\% y en tercer lugar RPM con un 12\%. La patología predominante fue ITU en ambos grupos etarios (Tabla № 6).

Asimismo, las gestantes adolescentes presentaron complicaciones en el parto siendo el primero de un Expulsivo prolongado y retención de restos placentarios con $17 \%$, segundo lugar trabajo de parto prolongado e inducción de parto con un $13 \%$ En las gestantes adultas en primer lugar fue conducción del parto en un $26 \%$, inducción del parto 19\% seguido de retención de restos placentarios $18 \%$ (Tabla № 7 ).

Por último se evidencia que las complicaciones neonatales de madres adolescentes en primer lugar es RCIU con un $35 \%$, en segundo lugar tenemos asfixia y prematurez con un $17 \%$ y en tercer lugar con un SDR. En las gestantes adultas en primer lugar RCIU con un $42 \%$, en segundo lugar Ictericia Neonatal con un $26 \%$ y en tercer lugar prematurez con un 18\%. En ambos grupos etarios la complicación más frecuente es RCIU (Tabla № 8)

TABLA № 01. DISTRIBUCIÓN DE GESTANTES SEGÚN EDAD ATENDIDAS EN EL HOSPITAL FÉLIX MAYORCA SOTO TARMA DE ABRIL - SEPTIEMBRE 2017

\begin{tabular}{ccc}
\hline EDAD & № & $\%$ \\
\hline $14-19$ & 23 & 11 \\
\hline $20-25$ & 53 & 26 \\
\hline $26-31$ & 66 & 33 \\
\hline $32-37$ & 44 & 22 \\
\hline $38-43$ & 16 & 8 \\
\hline TOTAL & $\mathbf{2 0 2}$ & $\mathbf{1 0 0}$
\end{tabular}

TABLA № 02. DISTRIBUCIÓN DE GESTANTES SEGÚN ESTADO CIVIL ATENDIDAS EN EL HOSPITAL FÉLIX MAYORCA SOTO TARMA DE ABRIL - SEPTIEMBRE 2017

\begin{tabular}{ccccccc}
\hline ESTADO CIVIL & ADOLESCENTES & $\%$ & ADULTAS & $\%$ & TOTAL & $\%$ \\
\hline SOLTERA & 17 & 74 & 18 & 10 & 35 & 17 \\
\hline CASADA & 0 & 0 & 40 & 22 & 40 & 20 \\
\hline CONVIVIENTE & 6 & 26 & 88 & 49 & 94 & 47 \\
\hline DIVORCIADA & 0 & 0 & 33 & 18 & 33 & 16 \\
\hline TOTAL & $\mathbf{2 3}$ & $\mathbf{1 0 0}$ & $\mathbf{1 7 9}$ & $\mathbf{1 0 0}$ & $\mathbf{2 0 2}$ & $\mathbf{1 0 0}$ \\
\hline
\end{tabular}


TABLA № 03. DISTRIBUCIÓN DE GESTANTES SEGÚN PARIDAD ATENDIDAS EN EL HOSPITAL FÉLIX MAYORCA SOTO TARMA DE ABRIL - SEPTIEMBRE 2017

\begin{tabular}{ccccccc}
\hline PARIDAD & ADOLESCENTE & $\%$ & ADULTA & $\%$ & TOTAL & $\%$ \\
\hline PRIMIGESTA & 17 & 74 & 36 & 20 & 53 & 26 \\
\hline MULTIGESTA & 6 & 26 & 143 & 80 & 149 & 74 \\
\hline TOTAL & $\mathbf{2 3}$ & $\mathbf{1 0 0}$ & $\mathbf{1 7 9}$ & $\mathbf{1 0 0}$ & $\mathbf{2 0 2}$ & $\mathbf{1 0 0}$
\end{tabular}

TABLA № 04. DISTRIBUCIÓN DE GESTANTES SEGÚN ATENCIÓN PRENATAL ATENDIDAS EN EL HOSPITAL FÉLIX MAYORCA SOTO TARMA DE ABRIL - SEPTIEMBRE 2017

\begin{tabular}{ccccccc}
\hline APN & ADOLESCENTES & $\%$ & ADULTAS & $\%$ & TOTAL & $\%$ \\
\hline 1 A 3 APN & 9 & 39 & 44 & 25 & 53 & 26 \\
\hline 2 A 6 APN & 11 & 48 & 100 & 56 & 111 & 55 \\
\hline 7 A MÁS APN & 3 & 13 & 35 & 20 & 38 & 19 \\
\hline TOTAL & $\mathbf{2 3}$ & $\mathbf{1 0 0}$ & $\mathbf{1 7 9}$ & $\mathbf{1 0 0}$ & $\mathbf{2 0 2}$ & $\mathbf{1 0 0}$
\end{tabular}

TABLA № 05. DISTRIBUCIÓN DE GESTANTES SEGÚN TIPO DE PARTO EN EL HOSPITAL FÉLIX MAYORCA SOTO TARMA DE ABRIL - SEPTIEMBRE 2017

\begin{tabular}{lcccccc}
\hline PARTO & ADOLESCENTES & $\%$ & ADULTAS & $\%$ & TOTAL & $\%$ \\
\hline EUTOCICO & 6 & 26 & 34 & 19 & 40 & 20 \\
\hline DISTOCICO VAGINAL & 9 & 39 & 88 & 49 & 97 & 48 \\
\hline CESAREA & 8 & 35 & 57 & 32 & 65 & 32 \\
\hline \multicolumn{1}{c}{ TOTAL } & $\mathbf{2 3}$ & $\mathbf{1 0 0}$ & $\mathbf{1 7 9}$ & 100 & $\mathbf{2 0 2}$ & $\mathbf{1 0 0}$ \\
\hline
\end{tabular}

TABLA № 06. DISTRIBUCIÓN DE GESTANTES SEGÚN TIPO DE COMPLICACIONES EN EL EMBARAZO EN EL HOSPITAL FÉLIX MAYORCA SOTO TARMA DE ABRIL - SEPTIEMBRE 2017

\begin{tabular}{lcccccc}
\hline COMPLICACIONES EMBARAZO & ADOLESCENTES & $\%$ & ADULTAS & $\%$ & TOTAL & $\%$ \\
\hline NINGUNO & 1 & 4 & 6 & 3 & 7 & 3 \\
\hline ABORTO & 2 & 9 & 13 & 7 & 15 & 7 \\
\hline HIPEREMESIS GRAVÍDICA & 2 & 9 & 9 & 5 & 11 & 5 \\
\hline APP & 3 & 13 & 5 & 3 & 8 & 4 \\
\hline ITU & 6 & 26 & 66 & 37 & 72 & 36 \\
\hline ANEMIA & 5 & 22 & 34 & 19 & 39 & 19 \\
\hline DPP & 0 & 0 & 2 & 1 & 2 & 1 \\
\hline PRE ECLAMPSIA & 2 & 9 & 6 & 3 & 8 & 4 \\
\hline ECLAMPSIA & 0 & & 3 & 2 & 3 & 1 \\
\hline RPM & 1 & 4 & 23 & 13 & 24 & 12 \\
\hline DISTOCIA DE PRESENTACIÓN & 1 & 4 & 12 & 7 & 13 & 6 \\
\hline \multicolumn{1}{c}{ TOTAL } & $\mathbf{2 3}$ & $\mathbf{1 0 0}$ & $\mathbf{1 7 9}$ & $\mathbf{1 0 0}$ & $\mathbf{2 0 2}$ & 100 \\
\hline
\end{tabular}

TABLA NNo 07. DISTRIBUCIÓN DE GESTANTES SEGÚN TIPO DE COMPLICACIONES EN EL PARTO EN EL HOSPITAL FÉLIX MAYORCA SOTO TARMA DE ABRIL - SEPTIEMBRE 2017

\begin{tabular}{lcccccc}
\hline \multicolumn{1}{c}{ COMPLICACIONES PARTO } & ADOLESCENTES & $\%$ & ADULTAS & $\%$ & TOTAL & $\%$ \\
\hline DCP & 2 & 9 & 8 & 4 & 10 & 5 \\
\hline TRABAJO PARTO PROLONGADO & 3 & 13 & 12 & 7 & 15 & 7 \\
\hline EXPULSIVO PROLONGADO & 4 & 17 & 12 & 7 & 15 & 7 \\
\hline PARTO PRECIPITADO & 2 & 9 & 8 & 4 & 10 & 5 \\
\hline DESGARROS PERINEALES & 1 & 4 & 9 & 5 & 10 & 5 \\
\hline DESGARROS DE CERVIX & 2 & 9 & 8 & 4 & 10 & 5 \\
\hline RETENCIÓN RESTOS PLACENTARIOS & 4 & 17 & 33 & 18 & 37 & 18 \\
\hline ATONIA UTERINA & 1 & 4 & 9 & 5 & 10 & 5 \\
\hline INDUCCIÓN PARTO & 1 & 4 & 34 & 19 & 35 & 17 \\
\hline CONDUCCIÓN DE PARTO & 3 & 13 & 46 & 26 & 49 & 24 \\
\hline \multicolumn{1}{c}{ TOTAL } & 23 & 100 & 179 & 100 & 202 & 100 \\
\hline
\end{tabular}


TABLA No 08 . DISTRIBUCIÓN DE COMPLICACIONES NEONATALES DE RECIEN NACIDOS ATENDIDOS EN EL HOSPITAL FÉLIX MAYORCA SOTO TARMA DE ABRIL - SEPTIEMBRE 2017

\begin{tabular}{lcccccc}
\hline $\begin{array}{c}\text { COMPLICACIONES } \\
\text { NEONATALES }\end{array}$ & ADOLESCENTES & $\%$ & ADULTAS & $\%$ & TOTAL & $\%$ \\
\hline SDR & 3 & 13 & 10 & 6 & 13 \\
\hline SEPSIS NEONATAL & 1 & 4 & 2 & 1 & 3 & 1 \\
\hline ASFIXIA PERINATAL & 4 & 17 & 5 & 3 & 9 & 4 \\
\hline ICTERICIA NEONATAL & 2 & 9 & 46 & 26 & 48 & 24 \\
\hline RCIU & 8 & 35 & 76 & 42 & 84 & 42 \\
\hline PREMATUREZ & 4 & 17 & 33 & 18 & 37 \\
\hline OBITO FETAL & 1 & 4 & 7 & 4 & 8 & 18 \\
\hline \multicolumn{1}{c}{ TOTAL } & $\mathbf{2 3}$ & $\mathbf{1 0 0}$ & $\mathbf{1 7 9}$ & 100 & $\mathbf{2 0 2}$ & 100 \\
\hline
\end{tabular}

\section{DISCUSIÓN}

El presente estudio describe las complicaciones maternas neonatales en gestantes adolescentes y adultas que acudieron al Hospital Félix Mayorca Soto durante los meses de marzo-agosto del 2017. Dentro de las características sociodemográficas se encuentra una edad media en las gestantes adolescentes de 14 19 con un $11 \%$, en las gestantes adultas se presentó un promedio de edad entre $26-31$ años, que representa un 33\% del total de las gestantes. En cuanto al estado civil se observa que el $74 \%$ de las adolescentes son solteras y en las gestantes adultas el $49 \%$ son convivientes. Heras y col. (2), encontraron que la gestantes de mayor edad presentaron más patologías asociadas al embarazo $29,2 \%$. Donoso (3), en su estudio demuestra que existe asociación entre bajo nivel socioeconómico y nutrición inadecuada con ruptura prematura de membranas (RPM); el presente estudio coincide con su investigación; al igual que con Guadamuz (4), quien refiere que el bajo nivel cultural y el bajo nivel socioeconómico están asociados a la RPM, aunque estos son dependientes uno del otro.

Respecto a la paridad en las gestantes adolescentes el $74 \%$ son primigestas, y en las gestantes adultas el $80 \%$ son multigestas. Según el número de atenciones prenatales, se encontró que el $48 \%$ de las gestantes adolecentes recibieron de 2 a 6 atenciones prenatales (APN) y en las gestantes adultas se encontró que un 56\% recibieron entre 2 a 6 APN. Con respecto al tipo de parto en las gestantes adolescentes se presentó un 39\% con parto vaginal distócico, en cuanto a las gestantes adultas $49 \%$ de parto vaginal distócico. Estas variables obstétricas coinciden con los datos encontrados en los estudios de Bustillos y col (5), quienes refieren haber encontrado en su población el mismo porcentaje de pacientes controladas (50\%). Gala (6), refiere haber encontrado porcentajes similares: $52 \%$ de pacientes controladas y 48 $\%$ no controladas.

Dentro de las complicaciones maternas, las complicaciones en las gestantes adolescentes se presentaron en mayor frecuencia las infecciones del tracto urinario en un $26 \%$ y las anemias en un $22 \%$. En las gestantes adultas las complicaciones de mayor frecuencia fueron ITU en un $72 \%$ y las anemias en un $39 \%$, estas fueron las de mayor frecuencia en las complicaciones las cuales coinciden con Bustillos y col, (5), quienes refieren similares datos en cuanto a complicaciones previas a una ruptura prematura de membranas (RPM), con infecciones transuretrales (ITU) de 33\% y anemia $47 \%$.

Dentro de las complicaciones en el parto en las gestantes adolecentes se presentaron las siguientes complicaciones en la misma proporción de $17 \%$ en expulsivo prolongado y retención de restos placentarios respectivamente. En las gestantes adultas se presentó conducción del parto en un $26 \%$ 
seguido de inducción de parto en un 19\%, lo mismo que difieren en el estudio realizado por Bravo y col (7). Hubo una mayor tasa de partos pretérminos que en la población general, el grupo de nulíparas tuvo una tasa de $21,7 \%$ sin diferencia significativa. Las mujeres multíparas tuvieron mayor número de neonatos macrosómicos $(11,3 \%)$, que el grupo de nulíparas $(p<0.05)$. Del mismo modo el estudio realizado por Bendezu (8) y Heras (2), consideran las patologías asociadas; la toxemia $(6,7 \%)$ la rotura prematura de membranas (RPM) (16,5\%) y, diabetes gestacional, metrorragia del primer trimestre, fueron mayores en las gestantes añosas respectivamente. Los productos macrosómicos y grandes para edad gestacional representaron $8,9 \%$ y $11,9 \%$, respectivamente, en el grupo en estudio, así como, los traumatismos obstétricos $(4,5 \%)$ y las hemorragias posparto $(10,1 \%)$. Concluye que en la gestación de mujeres de 35 años o mayores existe una elevada incidencia de gemelaridad, fetos macrosómicos y grandes para la edad gestacional, recién nacidos de bajo peso, cesárea, toxemia, RPM y hemorragia posparto.

En cuanto a las complicaciones neonatales se evidencia que en las gestantes adolescentes se presentó un $35 \%$ de casos de retardo de crecimiento intrauterino (RCIU), seguido de asfixia perinatal y prematurez en un $17 \%$ respectivamente. En las gestantes adultas se presentó un $42 \%$ de casos de RCIU, seguido de $26 \%$ de ictericia neonatal, el cual coincide con el estudio realizado por Ticona (9), quien refiere que la a tasa de mortalidad perinatal hospitalaria en el Perú en el 2000 fue 22,9/1000 nv; entre los factores de riesgo encontrados fue la edad materna 35 años a más $(\mathrm{OR}=1,6)$.

\section{CONCLUSIONES}

Las características comunes de la población materna del presente estudio fueron las siguientes: La edad promedio se registró en adolescentes de entre 14 - 19 años y en adultas de 26 a 31 años, el estado civil en adolescentes es de $74 \%$ solteras y en adultas $49 \%$ convivientes.

Se encontró que la población materna en su mayoría tuvieron atención prenatal entre 2 a 6 controles, en el caso de la paridad en las adolescentes en su mayoría fueron primigestas y en las gestantes adultas fueron multigestas, en el tipo de parto en ambos casos de la población terminaron con parto distócico vaginal.

Las complicaciones en el embarazo en ambos grupos adolescentes y adultas fueron ITU (Infecciones transuretrales) y Anemia, en las complicaciones del parto en las adolescentes fueron, expulsivo prolongado y retención de restos placentarios, en las gestantes adultas fueron conducción e inducción de parto y retención de restos placentarios.

Las complicaciones neonatales de gestantes adolescentes fueron de RCIU (Retardo de crecimiento intrauterino) y prematurez, en las gestantes adultas fueron de RCIU e Ictericia Neonatal.

\section{Correspondencia:}

Mg. Vilma Eneida Palpa Inga

Correo electrónico: eneidapalpa@gmail.com

\section{REFERENCIAS BIBLIOGRÁFICAS}

1. Fondo Mundial C. Niñas y adolescentes en América Latina y el Caribe. UNICEF. 2016; Asunto de Género N 133

2. Heras B, Gobernado J, Mora P, Almaraz A. La edad materna como factor de riesgo obstétrico. Resultado de edad avanzada como factor de riesgo. Servicio Obstétrico y Ginecológico. Hospital Clínico Valladolid, España. (Internet) Elsevier ;011 54(11):575-580 (Online) (Citado el 23 de Enero 2018 ) URL. Disponible en https: //scholar.google.com.pe 
3. Donoso E, Villarroel L. Edad materna avanzada y riesgo reproductivo.(Internet) Rev. Méd.de Chile scielo 2003;131(1):5559.(Online) (citado 25 de Enero 2018 ) URL disponible en: https://scielo.conicyt.cl/scielo.php?script=s ci arttext\&pid=S00349887200300010000 $\underline{8 \& \operatorname{lng}=\mathrm{es}}$

4. Guadamuz L. Nivel de Cumplimiento de Norma y Protocolo para la Atención de Ruptura Prematura de Membranas en embarazadas ingresadas en el servicio de maternidad del Hospital Gaspar García Laviana - Rivas, Enero 2014 a Octubre 2015. Monografía para Optar al título de Médico y Cirujano. Nicaragua.Facultad de Ciencias Médicas, Universidad Nacional Autonoma de Nicaragua Hospital Gaspar Garcia Laviana. Rivas; 2015.

5. Bustillos J, Giacomin L. Morbimortalidad Maternofetal en Embarazos de Mujeres Mayores de 35 Años.(Internert) Rev. Costa Rica. Cienc. Méd . 2004;25(1-2) (Online) (Citado 22 de Diciembre) URL. Disponible en: http://www.scielo.sa.cr/scielo.php?scri $\mathrm{pt}=$ sci arttext\&pid=S0253$29482004000100007 \& \operatorname{lng}=$ en

6. Gala H, Crespo E, García R, Bertrán J, Valón A. Factores de riesgo asociados al bajo peso al nacer en una comunidad venezolana.(Internet) Rev. MEDISAN.2010; 14(2) (Online) (citado el 22 de Diciembre 2017) URL. Disponible en: http://scielo.sld.cu/scielo.php?script= sci arttext\&pid=S1029301920100002000 $11 \& \operatorname{lng}=$ es.
7. Ventura. Cybertesis. Facultad de Medicina. Universidad Nacional Mayor de San Marcos. [Online].; 2004 [citado 2017 Diciembre 22. URL. Disponible en: http://repositorio.uwiener.edu.pe/bitstream /handle/123456789/47/017\%20EAP\%200 BSTETRICIA\%20TESIS\%20NUMERO\%2 03\%20BRAVO\%20\%26\%20ZAMBRANO. pdf ? sequence $=1$ \&isAllowed $=y$.

8. Bendezú G. Gestación en edad avanzada. (Internet) Rev. Peruana de Ginecologia y Obstetricia. 2001;47(3);166-170 (citado el 11 de Diciembre 2017) URL. Disponible en:

http://repebis.upch.edu.pe/cgibin/wxis.exe/ iah/? IsisScript=iah/iah. xis\&lang=E\&base=I ipecs\&nextAction=Ink\&exprSearch=CESA $\underline{\text { REA\&indexSearch }=\mathrm{MH}}$

9. Ticona M, Huanco D. Mortalidad Perinatal Hospitalaria en el Perú: Factores de Riesgo. (Internet) Rev. Chile. Obstet. Ginecol.2005; 70(5); 313-317 (Online) (citado EL 23 de Noviembre 2017 URL. Disponible en: https://scielo.conicyt.cl/ scielo.php?script=sci arttext\&pid=S07177 $\underline{5262005000500006 \& \operatorname{lng}=\mathrm{es} . .}$

Recibido: $30 / 06 / 2018$

Aprobado para Publicación: 21/10/2018 